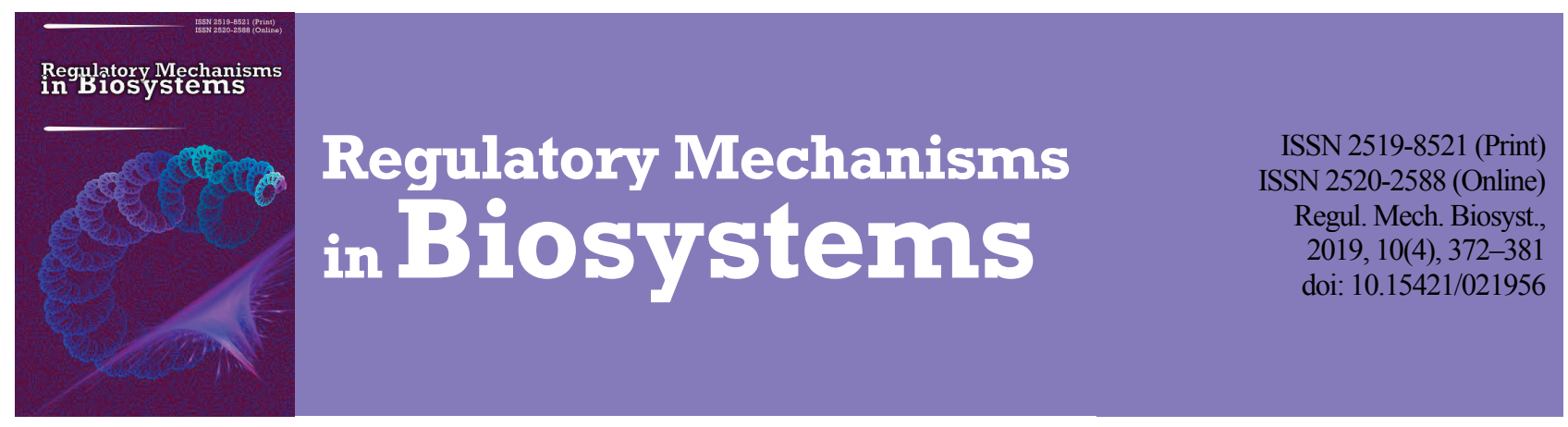

\title{
Impact of corvitin and alpha-ketoglutarate on heart morphology, expression and activity of matrix metalloproteinases $2 / 9$ in the heart of rats with doxorubicin-induced cardiomyopathy
}

\author{
I. A. Gordiienko, O. V. Poslavska, A. I. Shevtsova \\ SI “Dnipropetrovsk Medical Academy of the Ministry of Health of Ukraine”, Dnipro, Ukraine
}

Article info

Received 22.09.2019

Received in revised form 19.10.2019

Accepted 20.10.2019

SI "Dnipropetrovsk Medical Academy of the Ministry

of Health of Ukraine",

V. Vernadsky st., 9

Dnipro, 49044, Ukraine.

Tel. + 38-050-046-82-87.

E-mail:

gordienko.ju@gmail.com
Gordïenko, I. A., Poslavska, O. V., \& Shevtsova, A. I. (2019). Impact of corvitin and alpha-ketoglutarate on heart morphology, expression and activity of matrix metalloproteinases $2 / 9$ in the heart of rats with doxorubicin-induced cardiomyopathy. Regulatory Mechanisms in Biosystems, 10(4), 372-381. doi:10.15421/021956

The anthracycline anticancer drug doxorubicin is an effective and frequently used chemotherapeutic agent for various malignancies but it causes acute ventricular dysfunction, and also induces cardiomyopathy and heart failure. One of the mechanisms of cardiotoxicity of doxorubicin is oxidative stress, which stimulates myocardial remodeling. Matrix metalloproteinases MMP2 and MMP9 play a key role in this process. Despite extensive research, the expression and activity of these enzymes in the doxorubicindamaged heart and the effect of antioxidants on these indicators have not been sufficiently studied. The aim of this work was to study the possible cardioprotective effect of the antioxidant drugs corvitin and alpha-ketoglutarate in rats with doxorubicininduced cardiomyopathy. Cardiomyopathy in rats was induced by intraperitoneal administration of doxorubicin at the dose of $2.5 \mathrm{mg} / \mathrm{kg}$ body weight weekly for 28 days. Animals were divided into four groups: group 1 (control) received saline injections $(2.5 \mathrm{~mL} / \mathrm{kg})$; group 2 - injections of doxorubicin, 3 - corvitin $(5 \mathrm{mg} / \mathrm{kg}) 60$ minutes before doxorubicin administration, 4 - doxorubicin and $1 \%$ solution of alpha-ketoglutarate in drinking water ad libitum. Heart weight and shape indexes, the ratio of muscle to connective tissues, and heart histology were examined 7 days after the end of drug administration. Activity of MMP2 and MMP9, their intracellular distribution in myocardial tissues were evaluated by gelatin-zymography and immunohistochemistry. It was found that doxorubicin cardiomyopathy in rats was accompanied by a decrease in heart weight index, adaptive change of heart shape from ellipsoid to globular, increase of connective tissue content. Administration of doxorubicin results in profound lesion of the cardiomyocytes of the atria and ventricle, manifested by excessive cytoplasmic expression of MMP2 and MMP9 and an increase their activity in the heart. Antioxidants corvitin and alpha-ketoglutarate have insufficient regenerative effect on mass and shape indexes of heart however, exhibit potent cardioprotective effect by regulation of expression and activity of MMP2 and MMP9.

Keywords: gelatinases A and B; heart diseases; anthracycline antibiotic; antioxidants.

\section{Introduction}

Doxorubicin is one of the most effective anticancer drugs of the group of anthracycline antibiotics used to treat various types of cancers. However, its therapeutic utility is severely limited by its associated cardiotoxicity. Despite the enormous amount of research in this area, the exact molecular mechanisms underlying the toxic effects of doxorubicin on the heart are still an area that warrants further investigation. Analysis of the literature data suggests that the mechanisms of doxorubicin's cardiotoxicity are multifactorial (Renu et al., 2018; Wenningmann et al., 2019) and include the impairment of mitochondrial energetics, increase of reactive oxygen species (ROS) leading to oxidative stress, cell necrosis and induction of pro-apoptotic signaling pathways (Chaudhari et al., 2017; Pecoraro et al., 2017; Delehanty et al., 2018).

The most common manifestations of the cardiotoxicity of doxorubicin are cardiac dysfunction and dilated cardiomyopathy. Moreover, the degree of damage to the heart depends on the dose and duration of doxorubicin administration (Chatterjee et al., 2010; Loncar-Turukalo et al., 2015; Mancilla et al., 2019). An application of this drug was also found to influence sensitivity of the heart to ischemic injury. A recent study demonstrated that administration of doxorubicin upon induction of ischemia significantly increased the size of the infarction (Gharanei et al., 2013), but the exact mechanisms of these changes remains unclear. Doxorubicin-induced heart failure develops over a prolonged time 372 period and is associated with myocardial remodeling, which also involves changes in some structural proteins of the extracellular matrix. The matrix metalloproteinases (MMPs) play an important role in remodeling of the extracellular matrix. A special place for remodeling of the myocardium belongs to gelatinase A (MMP2) and gelatinase B or MMP9 (Polegato et al., 2015; Antonov et al., 2018). Due to their ability to hydrolyze collagen IV, which is the main component of the basal membranes, these MMP are involved in the development of numerous metabolic diseases, in processes of invasion, metastasis, vascularization/ angiogenesis and tissue remodeling (Hahn et al., 2014). That is why it is absolutely justified to study these enzymes in the heart of rats with doxorubicin-induced cardiomyopathy.

A number of potential cardioprotective drugs have been explored including anti-oxidants, free radical scavengers, renin-angiotensin-system antagonists, cardioselective beta-blockers and statins (Shaker et al., 2018; Wenningmann et al., 2019). Despite promising animal studies, clinical trials have yielded conflicting results (Razavi-Azarkhiavi et al., 2016; Cappetta et al., 2017). Recently, close attention has been paid to the bioflavonoid quercetin, which possesses various biological properties including anti-oxidative, anti-inflammatory, anti-coagulation, and antiradical activities. Quercetin has certain preventive actions in the treatment of cardiovascular diseases (Jin et al., 2012; Patel et al., 2018), its applications before ischemia or during reperfusion protect the myocardium from ischemia/reperfusion injury (Bartekova et al., 2015). How- 
ever, the effect of this drug on the heart remodeling has hardly been studied. It should be noted that poor intestinal solubility and absorption, as well as rapid neutralization after oral administration - the "flavonoid paradox" - limit the bioavailability of this flavonoid (Gonzales et al., 2017). Currently, corvitin, which is a water-soluble form of quercetin for intravenous injections, is used as a drug with a pronounced anti-ischemic, anti-stroke, and anti-infarction activity. Corvitin has a high bioavailability with a sufficiently low level of its toxicity (Gonzales et al., 2017).

Another drug that can prevent the development of doxorubicin-induced cardiomyopathy is alpha-ketoglutarate. This central metabolite of the Krebs cycle is involved in multiple metabolic cellular signaling pathways. It functions as an energy source, a precursor in the amino acid biosynthesis, a signaling molecule, as well as a regulator of epigenetic processes (Sinton et al., 2017). It regulates the activity of prolyl-4 hydroxylase, which controls the biosynthesis of collagen, a component of connective tissue, and influences the function of the hypoxia-inducible factor, an important transcription factor in development and progression of neoplasia (MacKenzie et al., 2017). The metabolic and extra-metabolic functions of alpha-ketoglutarate in the organism open many different fields for therapeutic interventions. The regulatory role of alpha-ketoglutarate and its structural analogues in carcinogenesis and possibilities of using it as an anticancer agent are now being discussed (Sultana et al., 2016; Zdzisinska et al., 2016). Despite significant advances in the study of the therapeutic effects of alpha-ketoglutarate, its influence on the processes of heart regeneration is not well understood.

The aim of this work was to study possible cardioprotective effects of corvitin and alpha-ketoglutarate in rats with doxorubicin-induced cardiomyopathy.

\section{Materials and methods}

Animals. The care and use of animals was conducted in compliance with the principles outlined in the current Guide to the Care and Use of Experimental Animals and in accordance with the ethical standards established by the Law of Ukraine No 3447-IV, dated 21.02.2006 "On the Protection of Animals from Cruelty". The protocol of the experiments on the animals was approved by the Local Ethics Review Committee on Animal Experiments in Dnipropetrovsk Medical Academy (Dnipro, Ukraine). Wistar male rats weighing $210 \pm 5 \mathrm{~g}$ were exposed to standard conditions, such as reverse $12 \mathrm{~h}$ light-dark cycle and provided with laboratory nutrition and water ad libitum.

Reagents. Bovine serum albumin (BSA), gelatin (Sigma, СШA); doxorubicin-KMP, thiopental-KMP (Public Joint Stock Company "Kyivmedpreparat", Ukraine); corvitin (Borshchahivskiy Chemical-pharmaceutical Plant, Ukraine); alpha-ketoglutarate (SGPlus, Sweden); 3,3diaminobenzidine tetrahydrochloride (visualization system LabVisison Quanto, TermoScientific); standard kits for determination of aspartate aminotransferase, lactate dehydrogenase, creatine kinase MB (Elitech diagnostics Seppim S.A.S., France); acrylamide/bis-acrylamide, electrophoresis protein molecular weight standards (Bio-Rad Lab, USA); MMP2/9, rabbit polyclonal antibodies to MMP2/9 (Sigma, USA), goat antibodies to rabbit IgG conjugated with horseradish peroxidase (HRPconjugate, Proteintech Group, USA).

Experimental protocol. Doxorubicin cardiomyopathy (DCMP) in rats was induced by intraperitoneal injections of the anthracycline antibiotic doxorubicin weekly during 28 days in dose $2.5 \mathrm{mg} / \mathrm{kg}$ of weight (Kapelko, 1990). The animals were randomly divided into four groups, ten rats in each group. The 1st group (control) received saline injections $(2.5 \mathrm{~mL} / \mathrm{kg})$; the 2nd group of animals underwent doxorubicin cardiomyopathy (DCMP), the 3rd group of rats received corvitin $(5 \mathrm{mg} / \mathrm{kg})$ 60 minutes before doxorubicin injection. The animals of the 4th group received DCMP and 1\% solution of alpha-ketoglutarate (AKG) in drinking water $(0.5 \% \mathrm{Ca}-\mathrm{AKG}+0.5 \% \mathrm{Na}-\mathrm{AKG})$ ad libitum. The rats consumed $40-43 \mathrm{~mL}$ of water per $1 \mathrm{~kg}$ of weight. By the end of experiment, euthanasia of rats was performed using sodium thiopental $(60 \mu \mathrm{g} / \mathrm{kg})$, the hearts and blood plasma were taken out and used for the subsequent research.

Cardiac morphometry. The extracted hearts were dried on paper filters, fixed in 10\% neutral formalin $10 \mathrm{~min}$ and then their weight and morphometric characteristics were determined (Nazarova et al., 2014). The morphometry of histological preparations of the ventricles and atria were performed with a point technique using a special transparent stereometric grid. The microscopic field of view was covered with a grid on which the points are located at the same distance from each other. The percentage of connective tissue to muscle was determined in ten fields of view by the ratio of the number of points that were overlaid on one or another type of tissue (Avtandilov, 2002).

The heart weight index was calculated as the ratio of the heart weight to the body weight of the experimental rats by the following formula:

$$
\mathrm{HWI}=(\mathrm{m} / \mathrm{M}) \times 100 \text {, }
$$

where HWI - heart weight index (\%), m - heart weight $(\mathrm{g})$ and $\mathrm{M}-$ body weight $(\mathrm{g})$ of experimental animals.

The heart shape index was determined as follows: $\mathrm{HSI}=(\mathrm{b} / \mathrm{l}) \times 100$,

where HSI - heart shape index $(\%), b$ - width of heart $(\mathrm{cm}), 1$ - length (cm) of heart. The shape of the heart at this index was evaluated as following: $<65 \%$ - conic, $65-75 \%$ - ellipsoidal, $>75 \%$ - globular.

Biochemical analysis. Plasma of experimental animals was used for biochemical analysis, where activity of aspartate aminotransferase (AST), lactate dehydrogenase (LDH) and MB-form of creatine kinase (CK-MB) was determined according to the instructions of commercial test-systems.

Histopathology and immunohistochemistry. The left part of the rat hearts fixed in $10 \%$ formalin was buffered in PBS (pH 7.4) for 24 hours at room temperature, dehydrated in increasing concentrations of isopropanol and embedded in paraffin. At least four $5 \mu \mathrm{m}$ thick slices of the left atrium and ventricle were obtained from each specimen for histological study of the structural changes in the myocardium. All sections were evaluated using light microscopy (Zeiss Primo Star microscope) at a magnification of $\times 400$.

The expression of MMP2 and MMP9 was evaluated by immunohistochemical analysis, using an indirect two-step method. The sections were deparaffinized with xylene, rehydrated with graded ethanol and washed in water. Then the sections were boiled in a microwave for 15 min for antigen retrieval, after which they were treated with $3 \%$ hydrogen peroxide for $15 \mathrm{~min}$ at room temperature to block endogenous peroxidase. The sites of nonspecific antibody binding were blocked by BSA-containing solution, after which the tissue sections were incubated with rabbit polyclonal antibodies (1:300) against MMP2 and MMP9 for $60 \mathrm{~min}$ at room temperature. Then, the sections were washed three times with phosphate-buffered saline (PBS) and incubated for $60 \mathrm{~min}$ with the HRP-conjugate at room temperature. The slices were washed by PBS again and exposed with 3,3-diaminobenzidine tetrachloride for $5 \mathrm{~min}$ at ambient temperature, washed in distilled water, post-stained with Mayer's hematoxylin. To quantify immunohistochemical expression, 7 to 10 fields in each slide (at a magnification of 400 times) were randomly chosen and colour reaction was evaluated (brown cytoplasmic staining). The results are presented as four different levels: $0-$ negative, 1 - weak, 2-moderate, and 3 -strong, reaction.

Fractionation of the soluble cardiac proteins. The fractions of soluble cardiac proteins were obtained by homogenization of the heart pieces in $0.025 \mathrm{M}$ tris- $\mathrm{HCl}$ buffer $\mathrm{pH} 7.2$ in the ratio $1: 4(\mathrm{~g} / \mathrm{mL})$, following centrifugation at $2860 \mathrm{~g}$ during $5 \mathrm{~min}$ at $4{ }^{\circ} \mathrm{C}$. The supernatants were sampled for further experiments. Protein concentrations in fractions of soluble cardiac proteins were determined by the Bradford (1976) method.

Gelatin-zymography. Activities of MMP2 and MMP9 were determined by the method of direct gelatin-zymography after vertical electrophoresis of the fractions of soluble cardiac proteins in $7.5 \%$ polyacrylamide gel containing $0.1 \%$ SDS and $1 \%$ gelatin. After electrophoresis, the gels washed twice for $20 \mathrm{~min}$ each with $50 \mathrm{mmol} / \mathrm{L}$ Tris- $\mathrm{HCl}$ (pH 7,4), containing 2.5\% Triton X-100, and then incubated 24 hours at $37{ }^{\circ} \mathrm{C}$ in a substrate buffer containing $50 \mathrm{mmol} / \mathrm{L}$ Tris- $\mathrm{HCl}, 10 \mathrm{mmol} / \mathrm{L}$ $\mathrm{CaCl}_{2}$ and $1.25 \%$ Triton $\mathrm{X}-100(\mathrm{pH} 7.4)$. After incubation, the gels were stained with 1\% Coomassie Brilliant Blue G-250 and then destained using the mix of $40 \%$ methanol and $10 \%$ acetic acid. The MMP were manifested as transparent bands against a dark blue background. In the following step, zymograms were converted into digital format and activity of MMPs calculated with the program Videodensitometer Sorbfil 2.0. The activities of MMP2 and MMP9 were evaluated in arbi- 
trary units $(\mathrm{AU})$ relative to their level in the standard sample, where the activity was taken as $1 \mathrm{AU}$. As a standard, we used a pool of cardiac fractions of the control group, which was obtained by mixing of the equal volumes of the fraction of soluble cardiac proteins in the control group (Shevcova et al., 2013). The aliquots of the standard were stored at $-80^{\circ} \mathrm{C}$. Specific activity of the studied enzymes in the hearts were calculated per $1 \mathrm{mg}$ of protein.

Statistical analysis. All data are presented as the means (x) and standard error (SE). Statistical analysis was performed using analysis of variance (one-way ANOVA) followed by the Tukey's multiple comparisons post hoc test. A value of $\mathrm{P}<0.05$ was considered as statistically significant.

\section{Results}

Physiological and biochemical parameters in plasma of the experimental rats. It was found that behavioural reactions of rats were suppressed after the doxorubicin injections due to deterioration of their physical and emotional states. Mortality in the experimental groups of animals was $15.6 \%$. The body weight of control group rats increased from $210.3 \pm 6.3 \mathrm{~g}$ at the beginning of the experiment to $229.3 \pm 11.8 \mathrm{~g}$ at the time of its completion. The weight of DCMP-rats had a tendency to decrease, it was only $93.8 \%$ by the end of the experiment compared with the start. A decrease of body weight by $16.2 \%$ observed in rats on the background of corvitin administration. The weight of the animals which consumed alpha-ketoglutarate with drinking water remained almost unchanged (Fig. 1).

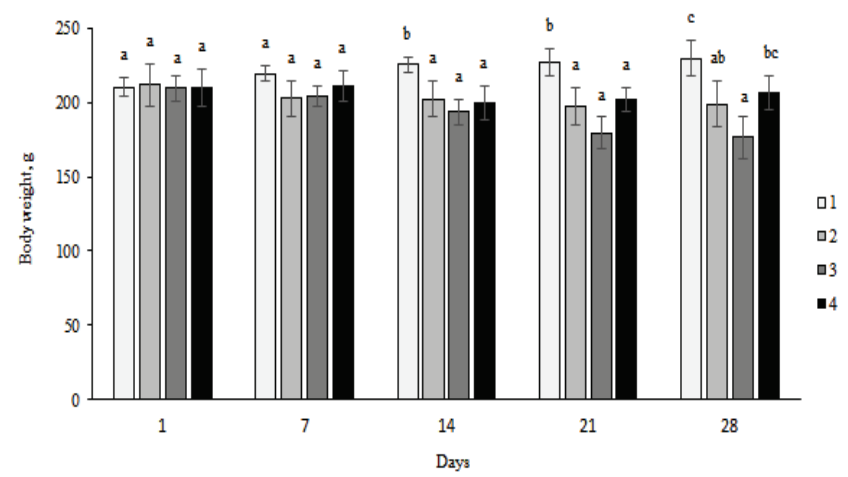

Fig. 1. The body weight ( $\mathrm{g}$ ) of rats during the experiment: 1 - control group, $2-\mathrm{DCMP}$ - group, 3 - DCMP + corvitin, 4-DCMP + alpha-ketoglutarate; DCMP - doxorubicin-induced cardiomyopathy; error bars represent means $\mathrm{x} \pm \mathrm{SE}$; statistical analysis was done by an one-way ANOVA with Tukey post hoc test

Biochemical studies were conducted to confirm doxorubicin-induced myocardial damage at the end of the experiment. The activities of aspartate aminotransferase (AST), lactate dehydrogenase (LDH) and MB-form of creatine kinase (CK-MB) were evaluated in the blood plasma of all animal groups (Table 1).

Table 1

The activity (IU/L) of aspartate aminotransferase (AST), lactate dehydrogenase (LDH) and MB-form of creatine kinase (CK-MB) in blood plasma of experimental rats ( $\mathrm{x} \pm \mathrm{SE}$, duration of experiment -28 days)

\begin{tabular}{lcccc}
\hline Parameter & $\begin{array}{c}\text { 1st group } \\
(\text { control, } \mathrm{n}=8)\end{array}$ & $\begin{array}{c}\text { 2nd group } \\
(\mathrm{DCMP}, \mathrm{n}=6)\end{array}$ & $\begin{array}{c}\text { 3rd group } \\
(\mathrm{DCMP}+ \\
\text { corvitin, } \mathrm{n}=7)\end{array}$ & $\begin{array}{c}\text { 4th group } \\
(\mathrm{DCMP}+ \\
\text { AKG, } \mathrm{n}=6)\end{array}$ \\
\hline AST & $145 \pm 13^{\mathrm{a}}$ & $184 \pm 4^{\mathrm{b}}$ & $136 \pm 9^{\mathrm{a}}$ & $158 \pm 6^{\mathrm{ab}}$ \\
LDH & $640 \pm 65^{\mathrm{ab}}$ & $1168 \pm 52^{\mathrm{c}}$ & $541 \pm 12^{\mathrm{a}}$ & $653 \pm 30^{\mathrm{b}}$ \\
CK-MB & $710 \pm 99^{\mathrm{a}}$ & $749 \pm 47^{\mathrm{a}}$ & $550 \pm 60^{\mathrm{a}}$ & $717 \pm 60^{\mathrm{a}}$ \\
\hline
\end{tabular}

Note: significant difference among groups is indicated by different letters; statistical analysis was done by a one-way ANOVA with Tukey's multiple comparisons post hoc test.

As follows from the presented data, the activity of all studied enzymes increased after the administration of doxorubicin, the most significant changes were found for LDH and AST. The activity of AST increased 1.3-fold and LDH - 1.8-fold in the DCMP-group relative to intact rats. In contrast to AST and LDH, the activity of the CK-MB in 2nd group of animals changed slightly.

It was found that the preventive use of corvitin or alpha-ketoglutarate had almost the same effect on the activity of the enzymes in rats with DCMP, both drugs significantly reduced the activity of AST and LDH in comparison with DCMP-group. It was also found that both the separate administration of doxorubicin and the use of this drug in combination with alpha-ketoglutarate did not significantly affect the activity of CK-MB, but the activity of this enzyme decreased by $26.6 \%$ after the administration of corvitin compared to the DCMP-group.

Impact of corvitin and alpha-ketoglutarate on morphological and histopathological parameters of the hearts. Morphometric characteristics, the heart weight (HWI) and shape (HSI) indexes were determined after pathoanatomical dissection of the animals. It was found that the HWI in rats of the DCMP-group decreased compared to the control group $(0.49 \pm 0.05 \%$ and $0.62 \pm 0.13 \%$, respectively) (Fig. 2a). The use of corvitin and alpha-ketoglutarate increased this index, but no complete recovery occurred. It should be noted that corvitin has a more pronounced restorative effect than alpha-ketoglutarate.
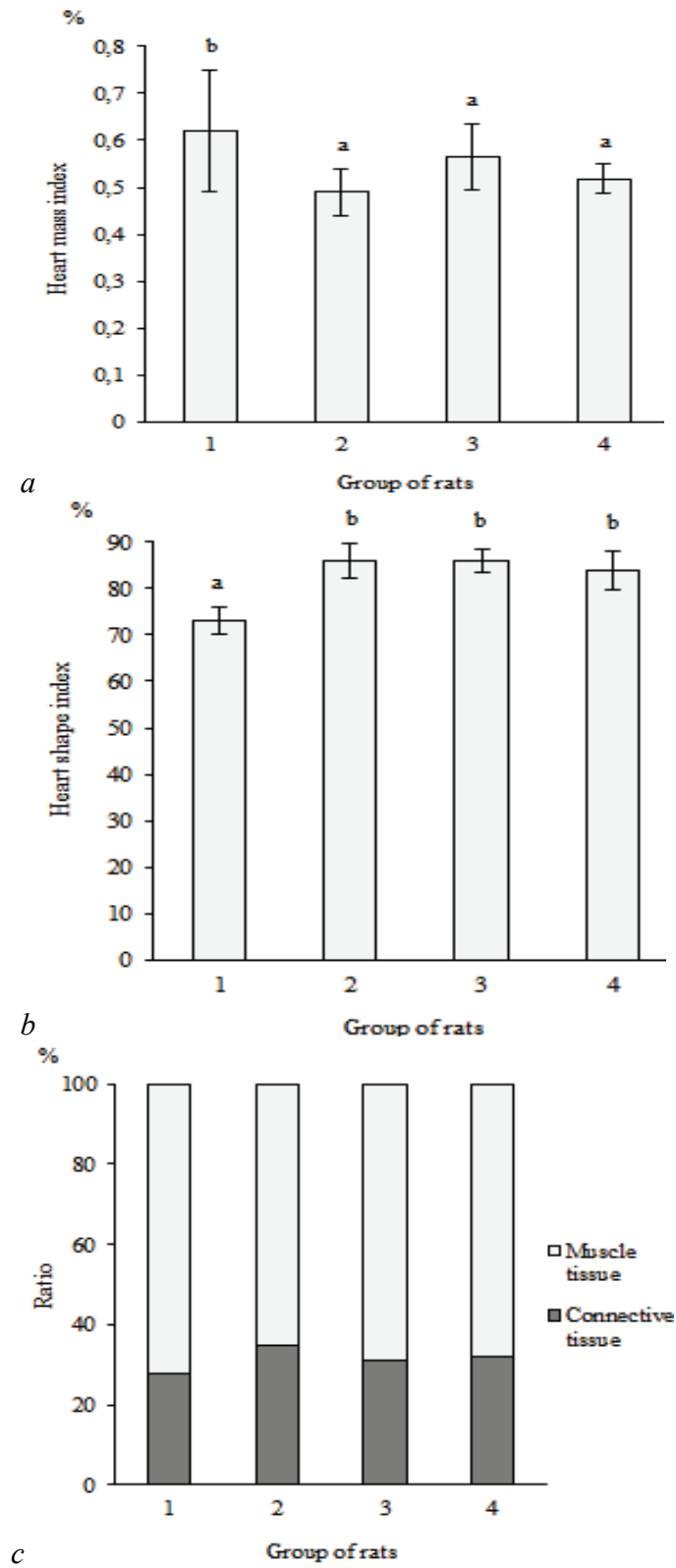

Fig. 2. Heart mass $(a)$ and heart shape $(b)$ indexes and the ratio of connective tissue to muscle tissue in heart $(c)$ of experimental groups of rats: see Fig. 1 
ATRIUM
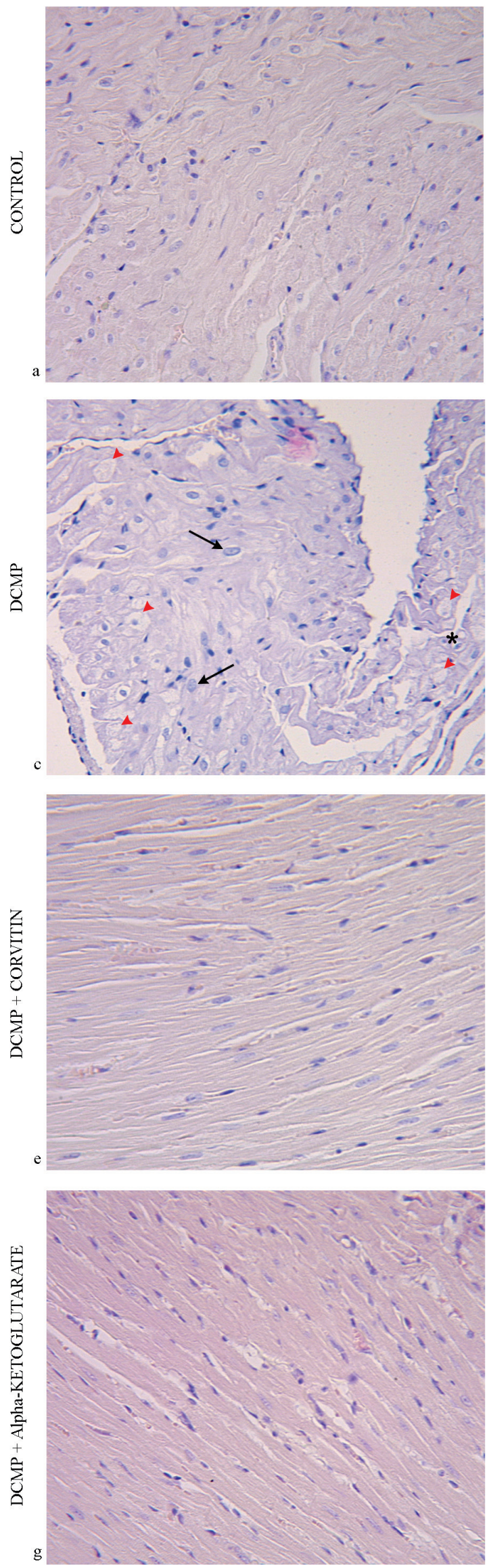

VENTRICLE
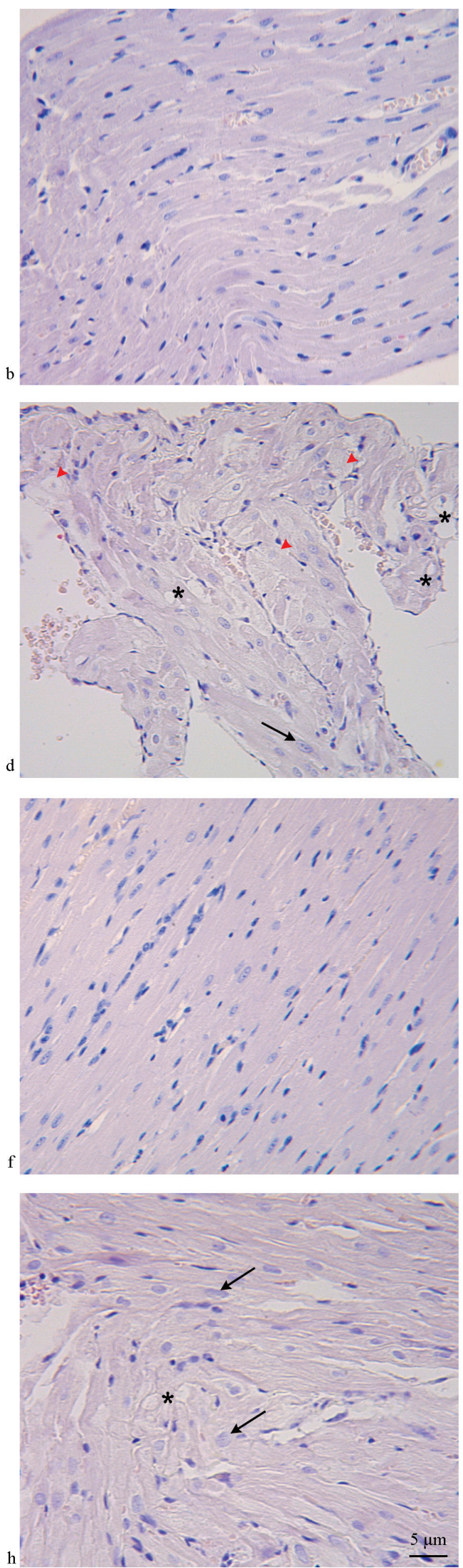

Fig. 3. Light micrographs of cardiac tissues of rats: control group $(a, b)$, group with doxorubicin-induced cardiomyopathy $(c, d)$, after administration of corvitin $(e, f)$ and alpha-ketoglutarate $(g, h)$; stain: Mayer's hematoxylin; Scale bar: $5 \mu \mathrm{m}$; black arrows indicate enlarged nuclei of cardiomyocytes with hypertrophy, red triangles - hydropic changes of the cytoplasm of cardiomyocytes with the phenomena of karyopicnosis or karyolysis, ${ }^{*}$ - fatty dystrophy 
Rats of the DCMP-group had a significant increase in HSI compared with intact animals ( $86.2 \pm 4.0 \%$ and $72.9 \pm 3.4 \%$, respectively), indicating a change in shape from ellipsoidal to globular. As can be seen from Figure $2 b$, neither corvitin nor alpha-ketoglutarate prevented these changes, although there was a slight tendency to decrease this index under the impact of alpha-ketoglutarate.

Analysis of the histological structure of the myocardium in the experimental groups allowed us to determine the ratio of connective (CT) and muscle (MT) tissues. It was shown that the total content of connective tissue in the hearts of control group animals was $28 \%$ and muscle $72 \%$ (Fig. 2c). The animals of the DCMP-group exhibited the decrease of muscle tissue content to $65 \%$ and increase of connective tissue content to $35 \%$. Calculation of the ratio of muscle tissue to the connective tissue in the heart revealed that the connective tissue component expands in the doxorubicin-injected rats with a corresponding decrease of muscle tissue: the ratio CT:MT changes from 0.38 in the control group to 0.53 in the DCMP-group. Under impact of corvitin the ratio CT:MT was 0.45 , and after the application of alpha-ketoglutarate -0.48 , which testifies to the cardioprotective effect of the drugs.

An in-depth analysis of the cellular structures of the left atrium and ventricle allowed us to identify some histological changes. Non-damaged cardiomyocytes with preserved striation and complete absence of fatty dystrophy were present in the heart tissues of the control group (Fig. 3a, b). Another pattern was observed in the specimens of heart tissues of the DCMP-rats: damaged cardiomyocytes alternated with preserved ones, the hypertrophy sites - with the atrophy sites. Damaged cells were characterized by a combination of hydropic and fatty changes, by disappearance of the striation. Islets of cardiomyocytes with signs of karyopycnosis, karyorexis, karyolysis, plasmolysis and the areas of diffuse cardiosclerosis were found in the left atrium and ventricle of these rats (Fig. 3c, d).

Administration of corvitin and alpha-ketoglutarate against the background of doxorubicin injections contributes to the improvement of myocardial ultrastructure: most cardiomyocytes remains non-damaged, almost complete absence of fatty dystrophy is shown, and the transverse striation of the heart muscle remains. Areas of cardiosclerosis in the histological specimens are located only around the vessels, where small areas of hypertrophy are found (Fig. 3e-h). However, some cells with signs of hydropic dystrophy are saved in case of treatment by alphaketoglutarate.

Immunohistochemical expression of MMP2/9 in heart tissues of rats. Expression of matrix metalloproteinases MMP2 and MMP9 in the atria and ventricle of experimental animals were investigated by immunohistochemical analysis (Fig. 4, 5). Careful analysis of immunohistochemical staining of cellular structures revealed certain differences in the number and localization of the above mentioned metalloproteinases in the studied heart specimens. Negative and weakly positive cytoplasmic expression of MMP2 (Fig. 4a) were detected in the ventricular tissue of the control group of rats. Areas with moderate MMP2 expression (Fig. 4b) presented in the atrium among cardiomyocytes with weak and negative expression. As for another proteinase - MMP9 - its expression and intracellular localization were similar to MMP2 although the level of MMP9 in the atrium was higher than in the ventricle.

An increase in cytoplasmic expression of MMP2 and MMP9 was found in cardiomyocytes of DCMP-group, where rats were administerred by doxorubicin for 4 weeks. Particularly intense immunohistochemical staining was observed in places of mosaic accumulation of cardiomyocytes with signs of dystrophy (Fig. 4, 5c, d). However, the designated enzymes were not detected in the areas of cardiosclerosis in the observed specimens. It should be noted a decrease in immunohistochemical staining of MMP2 and MMP9 in cytoplasm of the cells with signs of caryopycnosis and plasmolysis as well as the appearance of intranuclear staining instead cytoplasmic (Fig. 6). This result indicates a profound damage of the cells, and such histological staining is known as dot-like reaction.

A weak or negative staining of MMP2/9 in the cytoplasm of cardiomyocytes was detected in the atrium and ventricle of rats which were treated with corvitin on the background of doxorubicin injections. At the same time, an increase of the staining intensity in diffusely scattered cardiomyocytes with signs of hydropic dystrophy was found in some areas of the investigated hearts (Fig. 4, 5e, f).

The results of investigation of the immunohistochemistry of heart tissues in rats of group 4 that received alpha-ketoglutarate were similar to those of the corvitin group. As in case of treatment by corvitin, we found a poor staining for the presence of MMP2 and MMP9 in the cytoplasm of cardiomyocytes. The colour intensity increased to moderate in the cardiomyocytes which were located near the vessels. The areas of moderate cytoplasmic staining in cardiomyocytes with signs of hydropic changing were more common in the ventricle (Fig. 4, 5f, h) than in the atrial tissue (Fig. 4, 5e, g). In the ventricle, there was also a strong expression of MMP2 and MMP9 in single sites of accumulations of cardiomyocytes with fatty dystrophy, but such areas were much smaller than in samples of DCMP-group.

Activity of MMP2 and MMP9 in the heart of experimental rats. The activity of matrix metalloproteinases in fractions of soluble cardiac proteins was determined by gelatin-zymography in our modification with calculation of the relative activity of MMPs in arbitrary units in 1 mg protein (Table 2).

\section{Table 2}

Protein concentration and relative activity of MMP2 and MMP9 in fraction of soluble cardiac proteins ( $x \pm$ SE, duration of experiment -28 days)

\begin{tabular}{|c|c|c|c|c|c|}
\hline \multicolumn{2}{|c|}{ Parameter } & $\begin{array}{l}\text { 1st group } \\
\text { (control, } \\
\mathrm{n}=8 \text { ) }\end{array}$ & $\begin{array}{c}\text { 2nd group } \\
\text { (DCMP, } \\
n=6)\end{array}$ & $\begin{array}{c}\text { 3rd group } \\
\text { (DCMP+ } \\
\text { corvitin, } \\
n=7 \text { ) }\end{array}$ & $\begin{array}{c}\text { 4th group } \\
\text { (DCMP + } \\
\text { AKG, } \\
n=6)\end{array}$ \\
\hline \multicolumn{2}{|c|}{$\begin{array}{l}\text { Concentration } \\
\text { of protein, } \mathrm{mg} / \mathrm{mL}\end{array}$} & $\begin{array}{c}7.43 \pm \\
0.91^{\mathrm{a}}\end{array}$ & $\begin{array}{l}8.72 \pm \\
0.87^{\mathrm{ab}}\end{array}$ & $\begin{array}{c}10.02 \pm \\
0.30^{\mathrm{b}}\end{array}$ & $\begin{array}{c}8.08 \\
\pm 1.01^{\mathrm{ab}}\end{array}$ \\
\hline \multirow{3}{*}{ 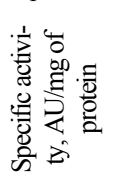 } & proMMP9 & $\begin{array}{c}14.04 \pm \\
0.99^{b}\end{array}$ & $\begin{array}{c}17.78 \pm \\
0.86^{\mathrm{b}}\end{array}$ & $\begin{array}{l}1.50 \pm \\
0.48^{\mathrm{a}}\end{array}$ & $\begin{array}{l}42.45 \\
\pm 1.87^{\mathrm{c}}\end{array}$ \\
\hline & proMMP2 & $\begin{array}{c}13.82 \pm \\
0.83^{\mathrm{ab}}\end{array}$ & $\begin{array}{c}17.55 \pm \\
0.79^{\mathrm{b}}\end{array}$ & $\begin{array}{c}8.38 \pm \\
0.29^{\mathrm{a}}\end{array}$ & $\begin{array}{c}40.72 \\
\pm 1.21^{\mathrm{c}}\end{array}$ \\
\hline & MMP2 & $\begin{array}{c}13.45 \pm \\
0.96^{\mathrm{b}}\end{array}$ & $\begin{array}{c}13.65 \pm \\
0.89^{\mathrm{b}}\end{array}$ & $\begin{array}{l}7.29 \pm \\
0.37^{\mathrm{a}}\end{array}$ & $\begin{array}{c}56.56 \\
\pm 1.63^{\mathrm{c}}\end{array}$ \\
\hline
\end{tabular}

Note: see Table 1.

As can be seen from the presented data, this method let us determine the proenzymatic forms of MMP2 and MMP9, as well as mature MMP2 in the heart. Active MMP9 was not determined on the zymograms under the conditions of our experiment (Fig. 7).

According to our results, the gelatinolytic activity of the investigated MMPs in the hearts of the control group was 13.45-14.04 AU/mg of protein. Their activity increased in the rats of the DCMP-group, especially significant changes occurred for proMMP2 and proMMP9. Corvitin caused significant inhibition of all forms of investigated MMPs in fractions of soluble cardiac proteins. Particularly pronounced changes were experienced by proMMP9, whose activity reduced 10-fold compared with the DCMP-group of rats. Less significant changes were found in activity of latent and mature MMP2, their activity decreased 2.1 and 1.9fold relative to the DCMP-group. The opposite effect was shown by alpha-ketoglutarate: the activity of the investigated MMPs was increased 2.3-4.1 fold in rats of this group in comparison with the DCMP-group.

\section{Discussion}

Cardiotoxicity of doxorubicin, which is currently used to treat many malignant tumours, including the various forms of leukemia, lymphoma, melanoma, uterine carcinomas, breast, and gastric cancers has been intensively discussed in recent years. Several mechanisms may play a role in the effects induced by this drug, including mitochondrial dysfunction, increased production of ROS, oxidative stress, DNA damage and apoptosis (Nebigil \& Desaubry, 2018). According to the ESMO Clinical Practice Guidelines, the consequences of doxorubicin administration in humans can be acute (occurring during or immediately after treatment), early (occurring within one year of exposure), and late (occurring one or more years after initial exposure) (Curigliano et al., 2012). Some strategies to prevent the cardiac toxicity of doxorubicin involve reducing dosages and prolonging the doxorubicin infusion time to limit its serum concentrations. 

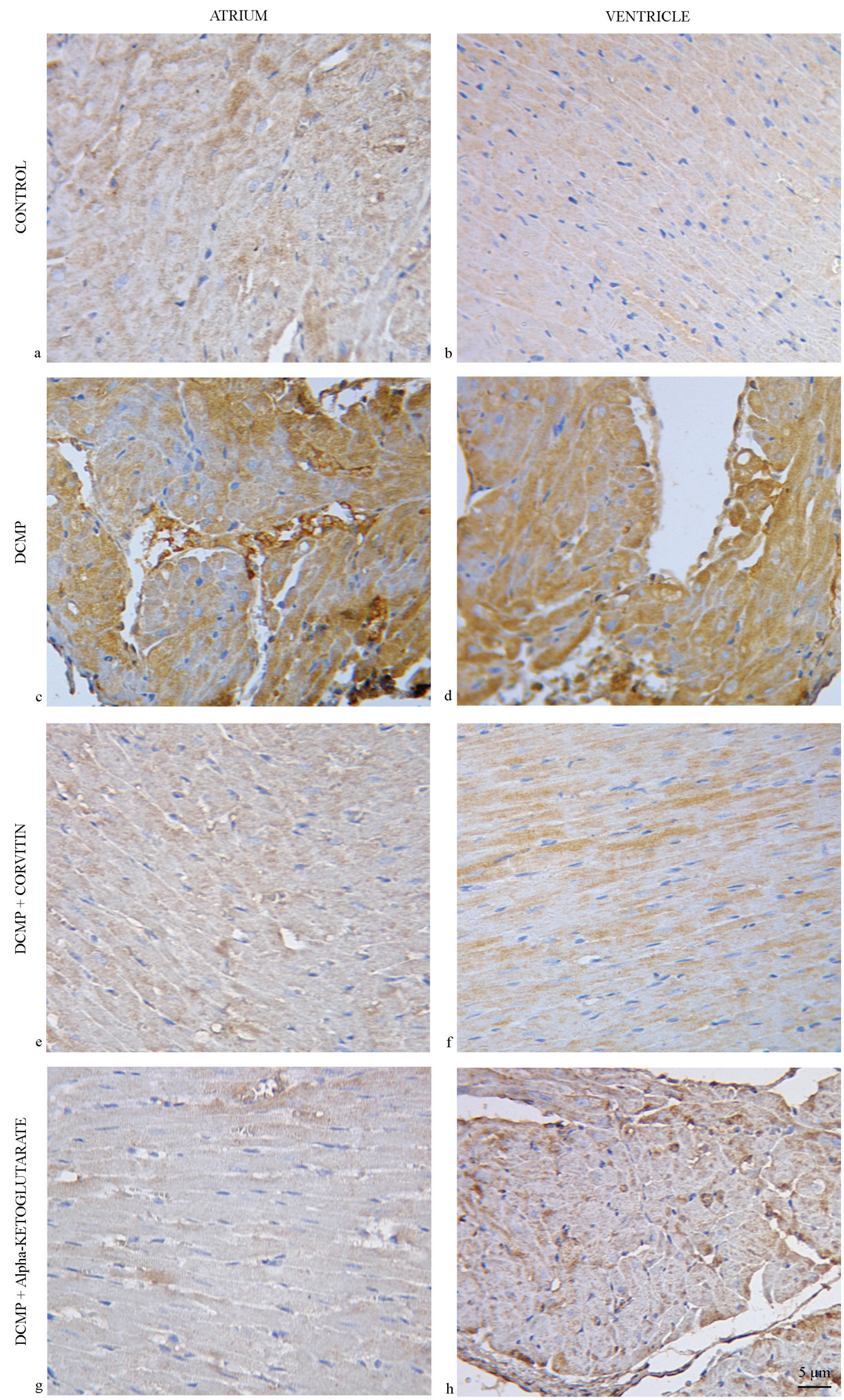

Fig. 4. Cytoplasmic expression of MMP2 in atrium and ventricle of control group $(a, b)$, group with doxorubicin-induced cardiomyopathy $(c, d)$, after administration of corvitin $(e, f)$ and alpha-ketoglutarate $(g, h)$; immunohistochemical method with Mayer's hematoxylin; scale bar: $5 \mu \mathrm{m}$ 

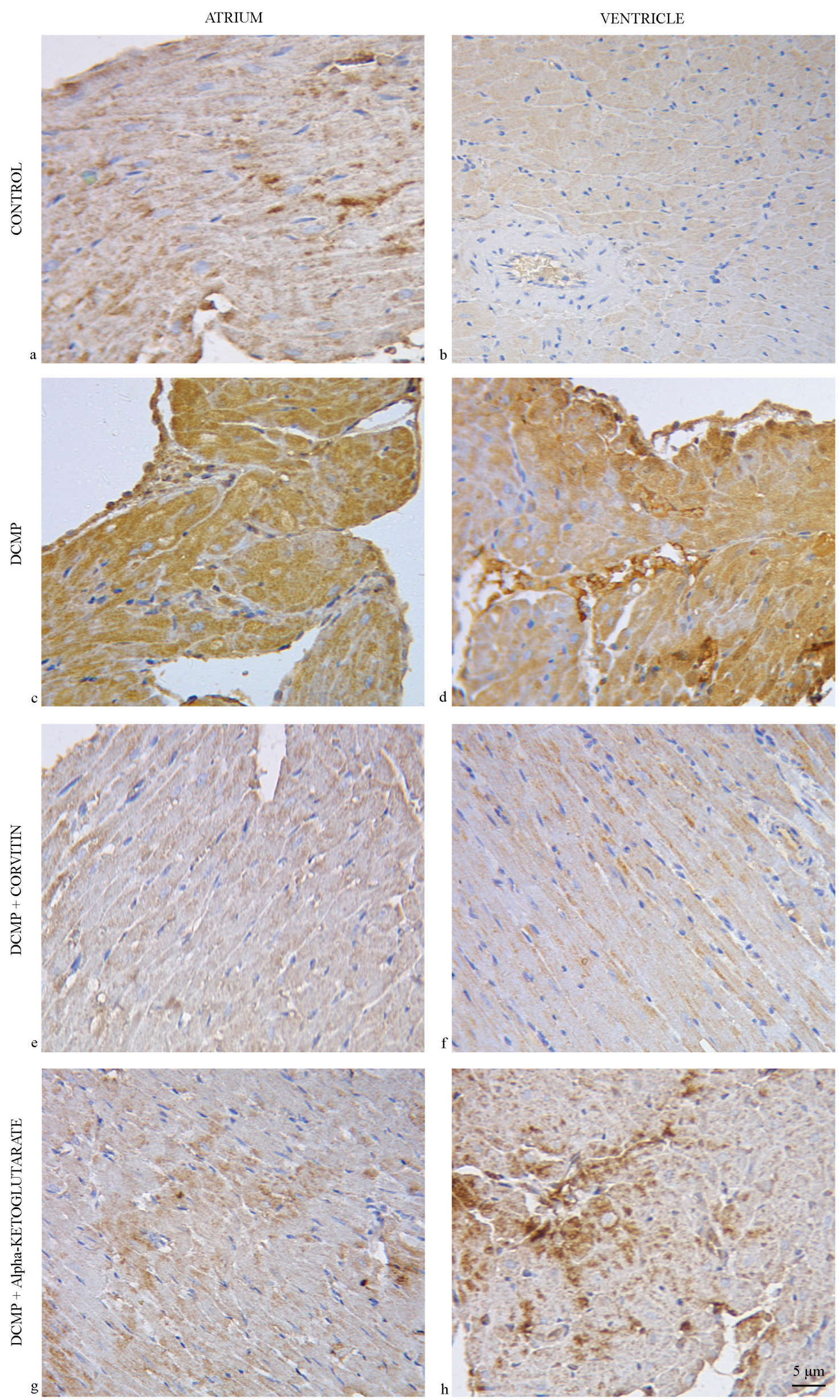

Fig. 5. Cytoplasmic expression of MMP9 in atrium and ventricle of experimental group of rats: see Fig. 4. 
In our animal model, we used repeated administration of lower doses of doxorubicin for four weeks, and we investigated the effects of corvitin and alpha-ketoglutarate on the physiological, morphological and histopathological parameters of the heart. Special attention was paid to the study of expression and activity of matrix metalloproteinases MMP2 and MMP9, which take a part in cardiac remodeling.

We have demonstrated that the repeated injections of doxorubicin (cumulative dose $10 \mathrm{mg} / \mathrm{kg}$ or $75 \mathrm{mg} / \mathrm{m}^{2}$ ) induced metabolic disorders, which led not only to the decrease of body weight but to significant changes of indexes of the heart weight and heart shape, as well as to histopathological changes in myocardium tissue of the experimental rats. According to the literature, the index of heart weight was almost unchanged after the introduction of doxorubicin at the dose of $15 \mathrm{mg} / \mathrm{kg}$ for 8 weeks (Kamel et al., 2017), while in our experiment this indicator decreased 1.3 times in comparison with the control group, despite the use of a 1.5 times lower cumulative dose of this drug. In addition, we have determined a tendency to change in heart shape from ellipsoidal to globular and decrease CT : MT. These findings are in consistent with studies showing that oxidative stress contributes to the 1.4 times decrease of heart weight index, change of heart geometry and development of its fibrosis (Angsutararux et al., 2015; Wang et al., 2019). Our histological findings indicate that the high risk of fibrosis under the influence of doxorubicin is caused by destructive-dystrophic changes in the left atrium and ventricle. This is supported by the fact that doxorubicin cardiotoxicity is manifested not only by structural disorders of cardiomyocytes but also by functional changes in cardiac fibroblasts. This antibiotic promotes cellular aging and induces differentiation of cardiac fibroblasts into the pro-fibrotic phenotype, myofibroblasts (Cappetta et al., 2016). In addition, doxorubicin-mediated interstitial and perivascular fibrosis occurs as a consequence of necrotic, apoptotic cell damage or pathological response to excessive ROS production (Carvalho et al., 2013, Zhan et al., 2016).

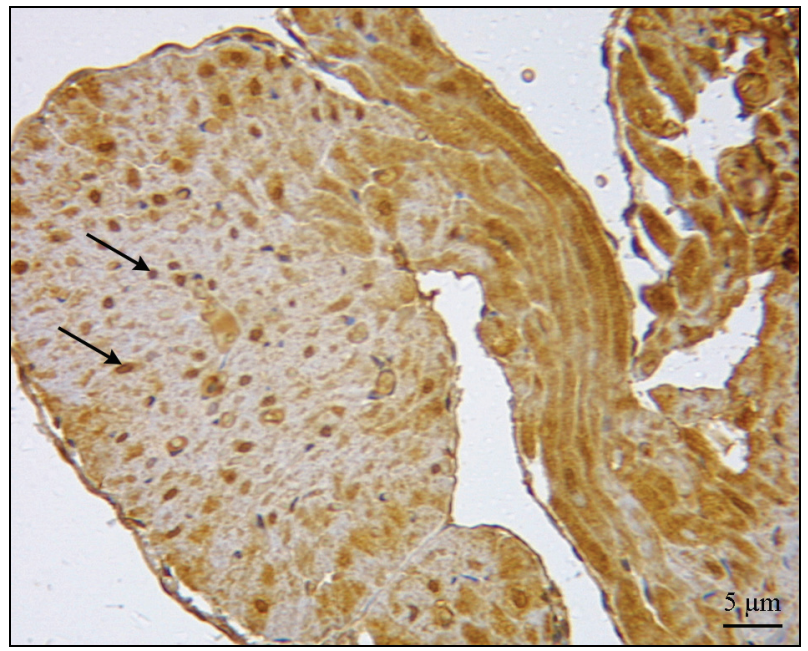

Fig. 6. Mixed cytoplasmic and intranuclear expression of MMP9 in the heart of a rat with doxorubicin-induced cardiomyopathy (dot-like phenomenon indicated by arrows): immunohistochemical method with Mayer's hematoxylin; scale bar: $5 \mu \mathrm{m}$

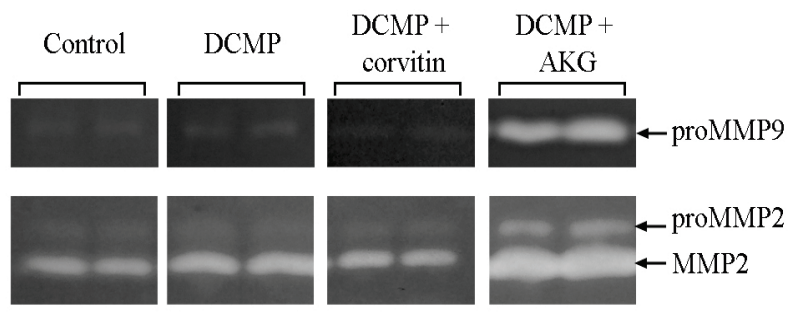

Fig. 7. Zymograms that illustrated the proteolytic bands of gelatinases: proMMP9 (92 kDa), proMMP2 (72 kDa), and active MMP2 (64 kDa); DCMP - doxorubicin-induced cardiomyopathy, $\mathrm{AKG}$ - alpha-ketoglutarate
Recent reports also indicated the key role of proteolytic enzymes in heart remodeling after doxorubicin treatment, especially matrix metalloproteinases, among them gelatinases A and B, or MMP2 and MMP9 are the most frequently discussed (Munch et al., 2016; Meschiari et al., 2017). These enzymes demonstrate substrate affinity for denatured collagen, basement membrane proteins such as collagen type IV, fibronectin, and laminin. MMP2 and MMP9 also exhibit proteolytic activity against elastin and proteoglycans. It was shown that the level of circulating gelatinases can serve as indicators of dilated cardiomyopathy (Pytliak et al., 2017), heart failure under doxorubicin treatment (Ivanova et al., 2012, Gordiienko et al., 2014), poor output of acute coronary syndrome (Lindsey et al., 2016) and efficiency of the therapy provided to heart failure patients (Radosinska et al., 2017). At the same time, the changes in expression and intracellular localization of these enzymes in heart tissue are poorly understood and discussed mostly as markers of post-infarction complications (Mayer et al., 2018). The results of our study indicate a profound cell damage, which is manifested by an increase in the cytoplasmic expression of MMP2 and MMP9 in most atrial and ventricular cardiomyocytes, an unspecific intranuclear dot-like reaction, and increase of the MMP2/9 activity in the fraction of cardiac proteins. The findings are consistent with the results of other researchers,who found an increased expression of both gelatinases in the left ventricle of rats with cyclophosphamide-induced cardiomyopathy (Koldysheva et al., 2018). This effect may be explained by the property of doxorubicin to cause oxidative stress through the activation of $\mathrm{NAD}(\mathrm{P}) \mathrm{H}$-oxidase and inducible NO-synthase. That results in the generation of a large number of ROS, followed by activation of redox-sensitive transcription factors NF-k $\beta / \mathrm{AP}-1$ and signaling cascades through G-protein, Ras and MAPK, as well as the release of a large number of proinflammatory cytokines and growth factors that synergistically stimulate overexpression of MMP2/9 (Octavia et al., 2012; Nakonato et al., 2015; Jung, et al., 2018). As was noted in our and other researches (Sinha et al., 2014), a certain amount of MMP2/9 has intranuclear localization. Some authors thought that these enzymes may be able to be involved in nucleus remodeling processes by degradation of some nuclear proteins such as transcription factors and RNA processing proteins (Lin et al., 2014).

At present, there are no specific clinical practice guidelines for the management of doxorubicin-induced cardiomyopathy. Overall, available cardioprotective measures include a combination of natural antioxidants, beta-blockers, angiotensin-converting-enzyme (ACE) inhibitors, angiotensin receptor blockers, diuretics, nitrates, and hydralazine (Wang et al., 2019; Wenningmann et al., 2019). Among those, antioxidants, ACE-inhibitors and beta-blockers showed the best cardioprotective results in patients under doxorubicin treatment (Curigliano et al., 2012; Kamel et al., 2017). However, additional clinical studies are necessary to develop alternative pharmacological approaches and clinical practices for the diversified patient population treated with anthracycline based chemotherapy. Taking into consideration the fact that oxidative stress and proteolysis play a significant role in development of doxorubicin cardiomyopathy, we have investigated the cardioprotective properties of corvitin and alpha-ketoglutarate, which exhibit a powerful antioxidant effect (Tkachenko et al., 2018). As follows from the presented results, both drugs have an insufficient regenerative effect on the morphological parameters of the heart - heart mass index and heart shape index (Fig. 2a, b), but show a strong effect on the expression and activity of MMP and MMP9 (Fig. 5, 6, Table 2). The decrease of gelatinases expression in the atria and ventricle, suppression of their activity in the heart muscle under the influence of corvitin may be explained from the standpoint of its antioxidant properties. The hydroxyl residues in the structure of the bioflavonoid quercetin, which is a main constituent of corvitin, may be a donor of hydrogen for free radicals, forming flavonoxyl radical. Such effect may determine quercetin's ability to restore superoxide dismutase activity, block NAD(P)H-oxidase, and enhance the synthesis of a tissue inhibitor of matrix metalloproteinases TIMP1, which impedes the activation of these MMPs (Wang et al., 2014). In addition, quercetin inhibits the synthesis of gene expression factor NF- $\kappa \mathrm{B}$, which leads to decrease in tumour necrosis factor TNF $\alpha$ and, indirectly, to decrease in gelatinases expression (Dong et al., 2014). Besides, quercetin is able to block PKC/ERK/AP1, MAP kinase and cJNK/AP signaling pathways, 
which also interfere with the expression of MMP2/9 (Wang et al., 2014). Unlike corvitin, the activity of the investigated MMPs is significantly increased with the simultaneous administration of doxorubicin and alpha-ketoglutarate. Probably, this effect is due not only to enhancement of the energy supply of myocardial cells, but also to the peculiarities of the composition of alpha-ketoglutarate. In our experiment we used the drug that contains the calcium and sodium salts of alpha-ketoglutarate. It is known that calcium ions are potent activators of matrix metalloproteinases both in vivo and in vitro. That is why $\mathrm{Ca}^{2+}$ is a necessary part of the incubation buffer during gelatin-zymography. Possibly, the prolonged administration of the calcium salt of alpha-ketoglutarate led to an increase in the activity of gelatinases.

\section{Conclusion}

The results of the study complement current views on the mechanisms of cardiotoxicity of the anthracycline antibiotic doxorubicin and provide new information about the cardioprotective effects of corvitin and alpha-ketoglutarate. The findings indicate that changes in MMP activity and expression occur with the action of even low doses of doxorubicin for 4 weeks. These changes are associated with impaired cardiomyocyte function, partial remodeling and fibrosis of the myocardial tissues. Antioxidants corvitin and alpha-ketoglutarate exert potent cardioprotective effects, regulating the expression and activity of matrix metalloproteinases MMP2 and MMP9.

The authors are grateful to Dr. M. Y. Zharikov for his assistance in the evaluation of morphological parameters and to Dr. Sci. Biol., Professor G. O. Ushakova for providing the alpha-ketoglutarate preparation for the research and for providing advisory assistance in discussing materials regarding this drug.

\section{References}

Angsutararux, P., Luanpitpong, S., \& Issaragrisil, S. (2015). Chemotherapy-induced cardiotoxicity: Overview of the roles of oxidative stress. Oxidative Medicine and Cellular Longevity, 2015, 795602.

Antonov, I. B., Kozlov, K. L., Paltseva, E. M., Polyakova, O. V., \& Linkova, N. S. (2018). Matrix metalloproteinases MMP-1 and MMP-9 and their inhibitor TIMP-1 as markers of dilated cardiomyopathy in patients of different age. Bulletin of Experimental Biology and Medicine, 164(4), 550-553.

Avtandilov, G. G. (Ed.). (2002). Osnovy kolichestvennoy patologicheskoy anatomii [Fundamentals of quantitative pathological anatomy]. Medicina, Moscow (in Russian).

Bartekova, M., Simoncikova, P., Fogarassyova, M., Ivanova, M., Okruhlicova, L., Tribulova, N., Dovinova, I., \& Barancik, M. (2015). Quercetin improves postischemic recovery of heart function in doxorubicin-treated rats and prevents doxorubicin-induced matrix metalloproteinase-2 activation and apoptosis induction. The International Journal of Molecular Sciences, 16(4), 81688185.

Bradford, M. M. (1976). A rapid and sensitive method for the quantification of microgramme quantities of protein utilizing the principle of protein-dye binding. Analytical Biochemistry, 72(1-2), 248-254.

Cappetta, D., Esposito, G., Coppini, R., Piegari, E., Russo, R., Ciuffreda, L. P., Rivellino, A., Santini, L., Rafaniello, C., Scavone, C., Rossi, F., Berrino, L., Urbanek, K., \& De Angelis, A. (2017). Effects of ranolazine in a model of doxorubicin-induced left ventricle diastolic dysfunction. The British Journal of Pharmacology, 174(21), 3696-3712.

Cappetta, D., Esposito, G., Piegari, E., Russo, R., Ciuffreda, L. P., Rivellino, A., Berrino, L., Rossi, F., De Angelis, A., \& Urbanek, K. (2016). SIRT1 activation attenuates diastolic dysfunction by reducing cardiac fibrosis in a model of anthracycline cardiomyopathy. The International Journal of Cardiology, 205, 99-110.

Carvalho, C. A. M., \& Thomazini, J. A. (2013). Morphometric and anatomical evaluation of the heart of Wistar rats. International Journal of Morphology, 31(2), 724-728.

Chatterjee, K., Zhang, J., Honbo, N., \& Karliner, J. S. (2010). Doxorubicin cardiomyopathy. Cardiology, 115(2), 155-162.

Chaudhari, U., Ellis, J. K., Wagh, V., Nemade, H., Hescheler, J., Keun, H. C., \& Sachinidis, A. (2017). Metabolite signatures of doxorubicin induced toxicity in human induced pluripotent stem cell-derived cardiomyocytes. Amino Acids, 49(12), 1955-1963.

Curigliano, G., Cardinale, D., Suter, T., Plataniotis, G., de Azambuja, E., Sandri, M. T., Criscitiello, C., Goldhirsch, A., Cipolla, C., \& Roila, F. (2012). Cardio- vascular toxicity induced by chemotherapy, targeted agents and radiotherapy: ESMO Clinical practice guidelines. Annals of Oncology, 23(7), 155-166.

Delehanty, J. B., Das, S., Goldberg, E., Sangtani, A., \& Knight, D. A. (2018). Synthesis of a reactive oxygen species responsive doxorubicin derivative. Molecules, 23(7), e1809.

Dong, Q, Chen, L., Lu, Q., Sharma, S., Li, L., Morimoto, S., \& Wang, G. (2014) Quercetin attenuates doxorubicin cardiotoxicity by modulating Bmi-1 expression. The British Journal of Pharmacology, 171(19), 4440-4454.

Gharanei, M., Hussain, A., Janneh, O., \& Maddock, H. L. (2013). Doxorubicin induced myocardial injury is exacerbated following ischaemic stress via opening of the mitochondrial permeability transition pore. Toxicology and Applied Pharmacology, 268, 149-156.

Gonzales, G. B. (2017). In vitro bioavailability and cellular bioactivity studies of flavonoids and flavonoid-rich plant extracts: Questions, considerations and future perspectives. Proceedings of the Nutrition Society, 76(3), 175-181.

Gordiienko, I. A., Babets, Y. V., Kulinich, A. O., Shevtsova, A. I., \& Ushakova, G. O. (2014). Activity of trypsin-like enzymes and gelatinases in rats with doxorubicin cardiomyopathy. The Ukrainian Biochemical Journal, 86(6), 139-146.

Hahn, V. S., Lenihan, D. J., \& Ky, B. (2014). Cancer therapy-induced cardiotoxicity: Basic mechanisms and potential cardioprotective therapies. Journal of the American Heart Association, 3(2), e000665.

Ivanova, M., Dovinova, I., Okruhlicova, L., Tribulova, N., Simoncíkova, P., Bartekova, M., Vlkovicova, J., \& Barancík, M. (2012). Chronic cardiotoxicity of doxorubicin involves activation of myocardial and circulating matrix metalloproteinases in rats. Acta Pharmacologica Sinica, 33(4), 459-469.

Jin, H. B., Yang, Y. B., Song, Y. L., Zhang, Y. C., \& Li, Y. R. (2012). Protective roles of quercetin in acute myocardial ischemia and reperfusion injury in rats. Molecular Biology Reports, 39(12), 11005-11009.

Jung, J.-S., Ahn, Y.-H., Moon, B.-I., \& Kim, H.-S. (2016). Exogenous $\mathrm{C}_{2}$ ceramide suppresses matrix metalloproteinase gene expression by inhibiting ROS production and MAPK signaling pathways in PMA-stimulated human astroglioma cells. The International Journal of Molecular Sciences, 17(4), 477.

Kamel, M. M., El-Farouk, L. O., Osman, A. S., Khorshid, O. A., \& ShabrawyAbdo, M. E. (2017). Comparative study of the protective effect of metformin and sitagliptin against doxorubicin-induced cardiotoxicity in rats. Clinical Pharmacology and Biopharmaceutics, 6, 174 .

Kapelko, V. I., \& Popovich, M. I. (Eds.). (1990). Metabolicheskie i funkcional'nye osnovy eksperimental'nyh kardiomiopatij [Metabolic and functional basis of experimental cardiomyopathies]. Stiinza, Kishinev (in Russian).

Kimura-Ohba, S., \& Yang, Y. (2016). Oxidative DNA damage mediated by intranuclear mmp activity is associated with neuronal apoptosis in ischemic stroke oxidative medicine and cellular longevity. Oxidative Medicine and Cellular Longevity, 2016, 6927328.

Koldysheva, E. V., Klinnikova, M. G., Nikityuk, D. B., Ivleva, E. K., Listvyagova, N. A., \& Lushnikova, E. L. (2018). Role of matrix metalloproteinase-2 in the development of cyclophosphamide-induced cardiomyopathy. Bulletin of Experimental Biology and Medicine, 164(4), 483-487.

Lin, H.-B., Cadete, V. J. J., Sra, B., Sawicka, J., Chen, Z., Bekar, L. K., Cayabyab, F., \& Sawicki, G. (2014). Inhibition of mmp-2 expression with sima increases baseline cardiomyocyte contractility and protects against simulated ischemic reperfusion injury. BioMed Research International, 2014, 810371.

Lindsey, M. L., Iyer, R. P., Jung, M., DeLeon-Pennell, K. Y., \& Ma, Y. (2016) Matrix metalloproteinases as input and output signals for post-myocardial infarction remodeling. Joumal of Molecular and Cellular Cardiology, 91, 134-140.

Loncar-Turukalo, T., Vasic M., Tasic, T., Mijatovic, G., Glumac, S., Bajic, D., \& Japunzic-Zigon, N. (2015). Heart rate dynamics in doxorubicin-induced cardiomyopathy. Physiological Measurement, 36(4), 727-739.

MacKenzie, E. D., Selak, M. A., Tennant, D. A., Payne, L. J., Crosby, S., Frederiksen, C. M., Watson, D. G., \& Gottlieb, E. (2007). Cell-permeating alphaketoglutarate derivatives alleviate pseudohypoxia in succinate dehydrogenasedeficient cells. Molecular and Cellular Biology, 27(9), 3282-3289.

Mancilla, T. R., Iskra, B., \& Aune, G. J. (2019). Doxorubicin-induced cardiomyopathy in children. Comprehensive Physiology, 9(3), 905-931.

Mayer, F., Falk, M., Huhn, R., Behmenburg, F., \& Ritz-Timme, S. (2018). Matrix metalloproteinases and tissue inhibitors of metalloproteinases: Immunhistochemical markers in the diagnosis of lethal myocardial infarctions? Forensic Science International, 288, 181-188.

Meschiari, C. A., Ero, O. K., Pan, H., Finkel, T., \& Lindsey, M. L. (2017). The impact of aging on cardiac extracellular matrix. Geroscience, 39(1), 7-18.

Munch, J., Avanesov, M., Bannas, P., Säring, D., Kramer, E., Mearini, G., Carrier, L., Suling, A., Lund, G., \& Patten, M. (2016). Serum matrix metalloproteinases as quantitative biomarkers for myocardial fibrosis and sudden cardiac death risk stratification in patients with hypertrophic cardiomyopathy. Journal of Cardiac Failure, 22(10), 845-850.

Nazarova, D. I., Kramar, S. B., Zharikov, M. Y., Kuznetsova, O. V., Kramar, G. Y., \& Kozhushko, V. V. (2014). The morphological characteristics of nutria heart. Morphologia, 8(1), 65-68. 
Nebigil, C. G., \& Desaubry, L. (2018). Updates in anthracycline-mediated cardiotoxicity. Frontiers in Pharmacology, 9, 1262.

Octavia, Y., Tocchetti, C. G., Gabrielson, C. L., Janssens, S., Crijns, H. J., \& Moens A. L. (2012). Doxorubicin-induced cardiomyopathy: From molecular mechanisms to therapeutic strategies. Joumal of Molecular and Cellular Cardiology, 52(6), 1213-1225.

Patel, R. V., Mistry, B. M., Shinde, S. K., Syed, R., Singh, V., \& Shin, H. S. (2018). Therapeutic potential of quercetin as a cardiovascular agent. The European Journal of Medicinal Chemistry, 155, 889-904.

Pecoraro, M., Rodriguez-Sinovas, A., Marzocco, S., Ciccarelli, M., Iaccarino, G., Pinto, A., \& Popolo, A. (2017). Cardiotoxic effects of short-term doxorubicin administration: Involvement of connexin 43 in calcium impairment. International Journal of Molecular Sciences, 18(10), e2121.

Polegato, B. F., Minicucci, M. F., Azevedo, P. S., Carvalho, R. F., Chiuso-Minicucci, F., Pereira, E. J., Paiva, S. A., Zornoff, L. A., Okoshi, M. P., Matsubara, B. B., \& Matsubara, L. S. (2015). Acute doxorubicin-induced cardiotoxicity is associated with matrix metalloproteinase-2 alterations in rats. Cellular Physiology and Biochemistry, 35(5), 1924-1933.

Pytliak, M., Vanik, V., \& Bojcik, P. (2017). Heart remodelation: Role of MMPs. The role of matrix metalloproteinase in human body pathologies.

Radosinska, J., Barancik, M., \& Vrbjar, N. (2017). Heart failure and role of circulating MMP-2 and MMP-9. Panminerva Medica, 59(3), 241-253.

Razavi-Azarkhiavi, K., Iranshahy, M., Sahebkar, A., Shirani, K., \& Karimi, G. (2016). The protective role of phenolic compounds against doxorubicin-induced cardiotoxicity: A comprehensive review. Nutrition and Cancer, 68(6), 892-917.

Renu, K., Abilash, V. G., Pichiah, T. P. B., \& Arunachalam, S. (2018). Molecular mechanism of doxorubicin-induced cardiomyopathy - an update. The European Journal of Pharmacology, 818, 241-253.

Shaker, R. A., Abboud, S. H., Assad, H. C., \& Hadi, N. (2018). Enoxaparin atenuates doxorubicin induced cardiotoxicity in rats via interfering with oxidative stress, inflammation and apoptosis. BMC Pharmacology and Toxicology, 19(1), 1-10.

Shevcova, A. I., Gordijenko, J. A., Shaul's'ka, O. E., \& Skoromna, A. S., (2013). Pat. 83196 UA, MPK G 01 N33/49 Sposib vyznachennja zhelatynaz u plazmi krovi [Method for determination of plasma gelatinases]; zajavnyk ta patentovlasnyk DZ 'Dnipropetrovs'ka medychna akademija MOZ Ukrai'ny”-zajavl. 26.03.13; opubl. 27.08.13, Bjul. № 16. - 4 p. (in Ukrainian).

Sinha, S. K., Asotra, K., Uzui, H., Nagwani, S., Mishra, V., \& Rajavashisth, T. B. (2014). Nuclear localization of catalytically active MMP-2 in endothelial cells and neurons. American Journal of Translational Research, 6(2), 155-162.

Sinton, M. C., Hay, D. C., \& Drake, A. J. (2019). Metabolic control of gene transcription in non-alcoholic fatty liver disease: The role of the epigenome. Clinical Epigenetics, 11(1), 1-12.

Sultana, S., Talegaonkar, S., Nishad, D. K., Mittal, G., Ahmad, F. J., \& Bhatnagar, A. (2018). Alpha ketoglutarate nanoparticles: A potentially effective treatment for cyanide poisoning. European Journal of Pharmaceutics and Biopharmaceutics, 126, 221-232.

Tkachenko, V., Kovalchuk, Y., Bondarenko, N., Bondarenko, O., Ushakova, G., \& Shevtsova, A. (2018). Effects of corvitin and 2-oxoglutarate on behavioral responses and antioxidant system in rats with pituitrin-isoproterenol-induced myocardial damage. Biochemistry Research International, 2018, 9302414.

Wang, L., Cheng, X., Li, H., Qiu, F., Yang, N., Wang, B., Lu, H., Wu, H., Shen, Y., Wang, Y., \& Jing, H. (2014). Quercetin reduces oxidative stress and inhibits activation of c Jun $\mathrm{N}$ terminal kinase/activator protein 1 signaling in an experimental mouse model of abdominal aortic aneurysm. Molecular Medicine Reports, 9(2), 435-442.

Wang, Q.-W., Yu, X.-F., Xu, H.-L., Zhao, X.-Z., \& Sui, D.-Y. (2019). Ginsenoside Re improves isoproterenol-induced myocardial fibrosis and heart failure in rats. Evidence-Based Complementary and Alternative Medicine, 2019, 3714508.

Wenningmann, N., Knapp, M., Ande, A., Vaidya, T. R., \& Ait-Oudhia, S. (2019). Insights into doxorubicin-induced cardiotoxicity: Molecular mechanisms, preventive strategies, and early monitoring. Molecular Pharmacology, 96(2), 219-232.

Zdzisinska, B., Zurek, A., \& Kandefer-Szerszen, M. (2017). Alpha-ketoglutarate as a molecule with pleiotropic activity: Well-known and novel possibilities of therapeutic use. Archivum Immunologiae et Therapiae Experimentalis, 65(1), 21-36.

Zhan, H., Aizawa, K., Sun, J., Tomida, S., Otsu, K., Conway, S. J., Mckinnon, P. J., Manabe, I., Komuro, I., Miyagawa, K., Nagai, R., \& Suzuki, T. (2016). Ataxia telangiectasia mutated in cardiac fibroblasts regulates doxorubicininduced cardiotoxicity. Cardiovascular Research, 110(1), 85-95. 